SUBmitted To Astrophysical Journal Letters

Preprint typeset using $\mathrm{LAT}_{\mathrm{E} X} \mathrm{X}$ style emulateapj v. 11/10/09

\title{
BIG FISH, SMALL FISH: TWO NEW ULTRA-FAINT SATELLITES OF THE MILKY WAY
}

\author{
V. Belokurov ${ }^{1}$, M. G. Walker ${ }^{1}$, N. W. Evans ${ }^{1}$, G. Gilmore ${ }^{1}$, M. J. Irwin ${ }^{1}$, D. Just ${ }^{2}$, S. Koposov$^{1}$, M. Mateo $^{3}$, E.

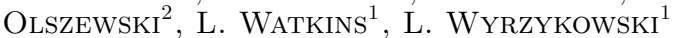 \\ SUBmitted to Astrophysical Journal Letters
}

\begin{abstract}
We report the discovery of two new Milky Way satellites in the neighboring constellations of Pisces and Pegasus identified in data from the Sloan Digital Sky Survey. Pisces II, an ultra-faint dwarf galaxy lies at the distance of $\sim 180 \mathrm{kpc}$, some $15^{\circ}$ away from the recently detected Pisces I. Segue 3, an ultra-faint star cluster lies at the distance of $16 \mathrm{kpc}$. We use deep follow-up imaging obtained with the 4-m Mayall telescope at Kitt Peak National Observatory to derive their structural parameters. Pisces II has a half-light radius of $\sim 60 \mathrm{pc}$, while Segue 3 is twenty times smaller at only 3pc.

Subject headings: galaxies: dwarf — galaxies: individual (Pisces, Pegasus) — Local Group
\end{abstract}

\section{INTRODUCTION}

Ultra-faint satellites of the Milky Way include dwarf galaxies (Willman et al. 2005; Zucker et al. 2006a, b; Belokurov et al. 2006, 2007a; Irwin et al. 2007; Belokurov et al. 2008) and star clusters (Koposov et al. 2007), as well as objects with intermediate properties (Walsh et al. 2007; Belokurov et al. 2009; Niederste-Ostholt et al. 2009). Defined by their extremely low surface brightness, these systems could only be detected with a massive multi-band imaging campaign like the Sloan Digital Sky Survey (SDSS).

In this Letter, we announce the discovery of two further Milky Way satellites in the Southern Galactic portion of the SDSS SEGUE survey. They lie in adjacent constellations and each extend only a couple of arc-minutes on the sky. However, their heliocentric distances differ by an order of magnitude, and, hence, so do their physical sizes. We name the dwarf galaxy in the constellation of Pisces, lying at the heliocentric distance of $\sim 180$ kpc and measuring $\sim 120 \mathrm{pc}$ across, Pisces II. This is the second Galactic stellar halo sub-structure in Pisces - the first, Pisces I was announced earlier this year by Watkins et al. (2009). Pisces I is much closer and more dispersed on the sky: it is at least several degrees across and lies at $\sim 80 \mathrm{kpc}$. Our second discovery is a feeble cluster of stars in the constellation of Pegasus. It has a half-light radius of $3 \mathrm{pc}$ and lies at a heliocentric distance of $16 \mathrm{kpc}$. We name it Segue 3, after SEGUE, the imaging survey in the data of which it was found.

In the analysis presented in this Letter we have extinction-corrected all magnitudes using the maps of Schlegel et al. (1998).

\section{DATA AND DISCOVERY}

The SDSS imaging data are available through the latest Data Release 7 (DR7) in two parts: i) $\sim 8000$ square degrees of the main SDSS field of view, mostly

\footnotetext{
${ }^{1}$ Institute of Astronomy, University of Cambridge, Madingley Road, Cambridge CB3 0HA,

UK; vasily, walker, nwe@ast. cam.ac.uk

2 Steward Observatory, University of Arizona, Tucson, AZ 85721, USA

${ }^{3}$ Department of Astronomy, University of Michigan, Ann Arbor, MI 48109, USA
}

TABLE 1

Properties of Pisces II And Segue 3

\begin{tabular}{lcc}
\hline \hline \multicolumn{1}{c}{ Parameter } & Pisces II & Segue 3 \\
\hline RA (J2000) & $22: 58: 31 \pm 6$ & $21: 21: 31 \pm 4$ \\
Dec (J2000) & $+05: 57: 09 \pm 4$ & $+19: 07: 02 \pm 4$ \\
Galactic $\ell$ & $79.21^{\circ}$ & $69.4^{\circ}$ \\
Galactic $b$ & $-47.11^{\circ}$ & $-21.27^{\circ}$ \\
$r_{h}$ (Plummer) & $1 !^{\prime} 1 \pm 0.1$ & $0.65 \pm 0.1$ \\
$\theta$ & $77^{\circ} \pm 12^{\circ}$ & $215^{\circ} \pm 20^{\circ}$ \\
$e$ & $0.4 \pm 0.1$ & $0.3 \pm 0.2$ \\
$(\mathrm{~m}-\mathrm{M})_{0}$ & $21^{\mathrm{m}} 3$ & $16^{\mathrm{m}} \cdot 1$ \\
$\mathrm{M}_{\text {tot }, \mathrm{V}}$ & $-5^{\mathrm{m}} 0$ & $-1^{\mathrm{m}} \cdot 2$ \\
\hline
\end{tabular}

${ }^{*}$ Magnitudes are accurate to $\sim \pm 0 .{ }^{\mathrm{m}} 5$ and are corrected for the Galactic foreground reddening.

around the North Galactic Cap and ii) 3000 square degrees of SEGUE imaging at low Galactic latitudes, with large portions of the Southern Galactic sky covered (Yanny et al. 2009). These imaging data are produced in five photometric bands, namely $u, g, r, i$, and $z$ and are automatically processed through the same pipelines to measure photometric and astrometric properties (Abazajian et al. 2009). The two imaging datasets differ in the continuity of the sky coverage and the amount of Galactic reddening: while most of the SDSS has contiguous coverage and is observed through minimal amounts of dust, SEGUE consists of tens of long, $2.5^{\circ}$-wide stripes affected by various amounts of Galactic extinction.

Both discontinuity in coverage and variable extinction complicate the search for stellar over-densities by adding non-Poissonian noise to the stellar density field. Nonetheless, applying our over-density detection algorithm (Belokurov et al. 2006) to the SEGUE data immediately yielded several promising candidates, which we are continuing to follow up with deep imaging and spectroscopy. We have already presented the first result, the discovery of the Segue 2 satellite (Belokurov et al. 2009). Several more candidate objects were detected at similar significance level. Pisces II was selected for follow-up, as it showed tentative evidence for the presence of Blue Horizontal Branch (BHB) stars. The case for Segue 3 was simpler: the object could actually be seen on the SDSS images. 

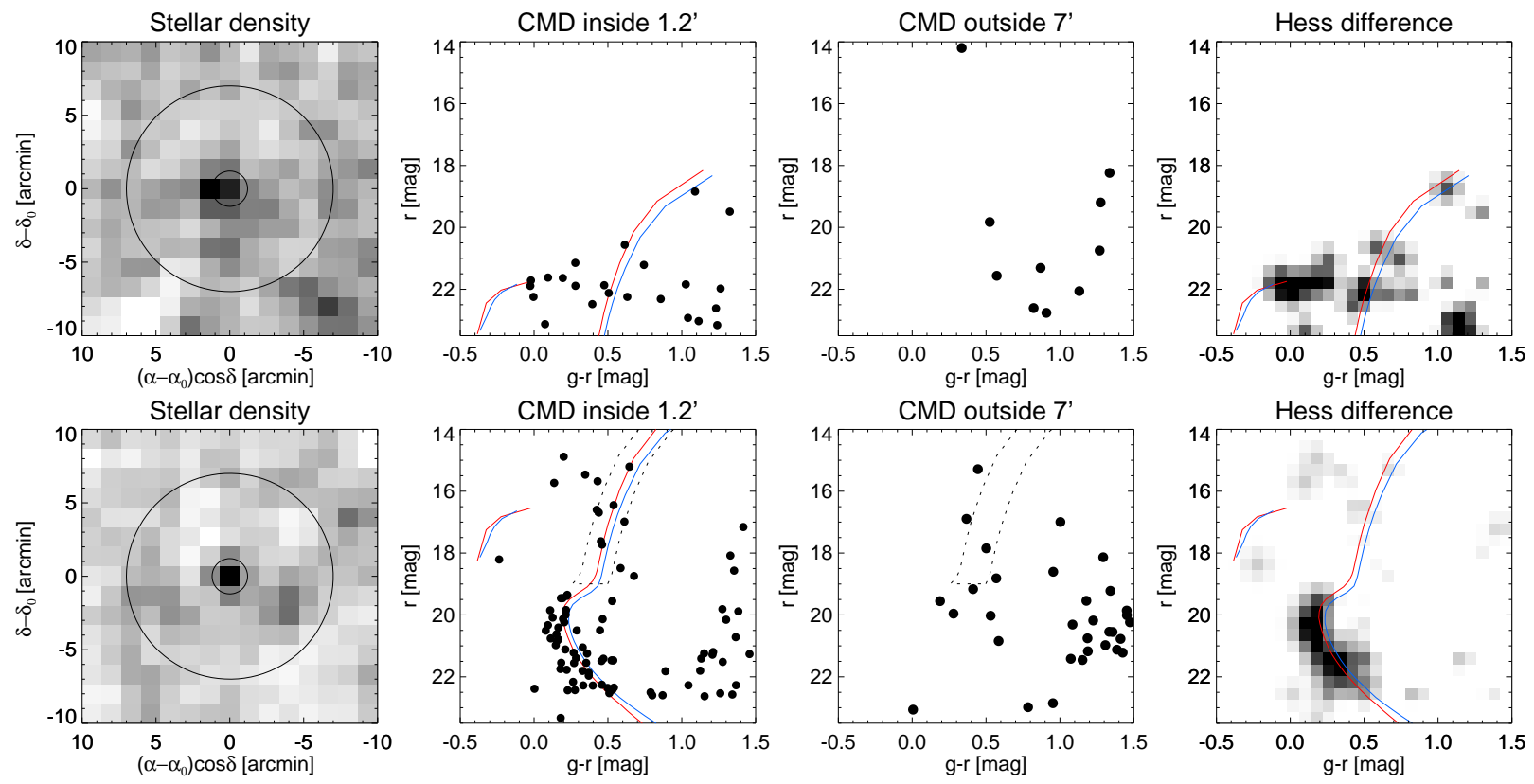

FIG. 1. - The SDSS view of Pisces II (upper row) and Segue 3 (lower row): Left: Density of stars in the SDSS catalogue centered on the object. The stars in the $10^{\prime} \times 10^{\prime}$ area are binned into $15 \times 15$ bins and smoothed with a Gaussian with FWHM of 1.5 pixel. Middle Left: CMD of all stars in a circle of radius $1 ! 2$ (marked on the left panel), dominated by the satellite's members. Middle Right: Comparison CMD of stars within the annulus $7^{\prime}$ to $7 ! 1$ showing the foreground. Right: Difference in Hess Diagrams. Pisces II populations (top right) can be hinted at by the red giant branch and blue horizontal branch. Segue 3 (bottom right) shows an obvious main sequence. Ridge-lines of M92 (red, $[\mathrm{Fe} / \mathrm{H}] \sim-2.3$ ) and M13 (blue, $[\mathrm{Fe} / \mathrm{H}] \sim-1.55$ ) are over-plotted. For Segue 3, we also show a mask built using M92 ridge-line to select possible red giant stars for luminosity calculation (lower middle left panel).

Fig. 1 shows the view of Pisces II and Segue 3 as seen by SDSS. The first of the four panels shows the density of all objects classified as stars by the SDSS pipeline down to $r=23$. In each case, there is a visible over-density at the center: Pisces II is detected with significance (Koposov et al. 2008) of $\sim 5$ and Segue 3 with significance of $\sim 7$. The next two panels are the color-magnitude diagrams (CMDs) of the object and the Galactic foreground around it. It requires a lot of imagination to see the red giant branch or indeed the blue horizontal branch of Pisces II. However, the Hess difference in the right panel of Fig. 1 shows that there are over-densities of blue-ish and reddish stars that can be interpreted as BHBs and RGBs at the heliocentric distance of $\sim 180 \mathrm{kpc}$. The lower panel of Fig. 1 presents the SDSS data for Segue 3, which is clearly a simpler case: the main sequence (MS) at $\sim 15 \mathrm{kpc}$ is obvious. It is, however, more difficult to identify the RGB population in Segue 3 as there is no obvious over-density in the Hess difference plot. In the absence of spectroscopic data, we gauge the likely membership by placing a mask that selects six potential members, at least one of which probably belong to the Galactic foreground. There is also a lone blue star in Segue 3, which looks slightly too faint to be classified as a BHB unambiguously.

\section{FOLLOW-UP IMAGING AND STRUCTURAL PARAMETERS}

During the nights of 19-24 September 2009, we obtained follow-up photometry of Pisces II and Segue 3 using the MOSAIC camera at the 4-m Mayall Telescope at Kitt Peak National Observatory, Arizona. For both objects, we observed a single $36^{\prime} \times 36^{\prime}$ field for $3 \times 900$ seconds in both $g$ and $r$ filters. Each of MOSAIC's eight detectors have $2048 \times 4096$ pixels with scale $0.258^{\prime \prime}$ per pixel (unbinned). For this run we enjoyed photometric conditions and typical seeing of $\lesssim 1^{\prime \prime}$. We processed raw MOSAIC frames using the set of IRAF-based procedures that have been developed for the NOAO Deep Wide-Field Surveyt (Jannuzi et al. 2000). These procedures include steps to correct for a pupil ghost that is caused by reflections off the atmospheric dispersion corrector. Briefly, we used averaged dome flats to generate a template image of the pupil ghost, which we then removed from the master flat field image. After dividing science frames by the master flat field, we then scaled and subtracted the pupil template image from science frames one by one. Visual inspection confirms that these steps successfully removed the pupil ghost from our science frames. Data stacking and the production of catalogues was performed using a general purpose pipeline for processing wide-field optical CCD data (Irwin \& Lewis 2001). For each image frame, an object catalog was generated and used to update the world coordinate system prior to stacking each set of 3 frames. A final set of object catalogs was generated from the stacked images and objects were morphologically classified as stellar or nonstellar (or noise-like). The detected objects in each passband were then merged by positional coincidence (within $1^{\prime \prime}$ ) to form a combined $g, r$ catalogue and photometrically calibrated on the SDSS system using stars in common.

4 Details of these reduction procedures are available at http://www.noao.edu/noao/noaodeep/ReductionOpt/frames.html 

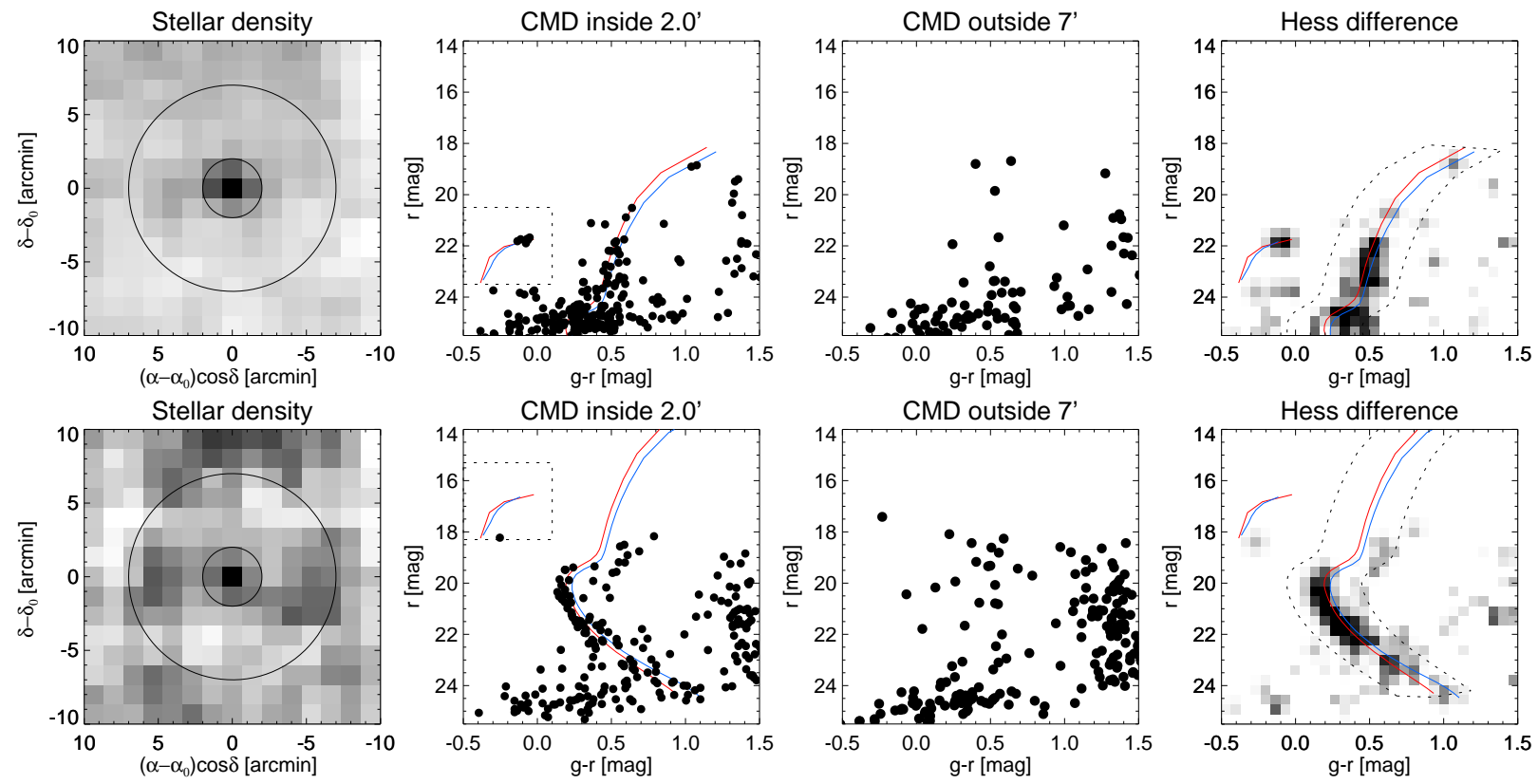

FIG. 2.- The KPNO view of Pisces II (upper row) and Segue 3 (lower row). The panels are the same as in Figure 1, with the inner radius increased to $2^{\prime}$. The KPNO saturation limit is fainter than that of SDSS at $r \sim 18$, but it reaches $\sim 2$ magnitudes deeper. The CMD of Pisces II shows the most obvious improvement. In the KPNO data, strong RGB and clear BHB and blue straggler populations can now be seen. The dotted box in the middle left is used to pick out the likely BHB members. The dotted lines in the right panel outline the regions used to select the satellite members.
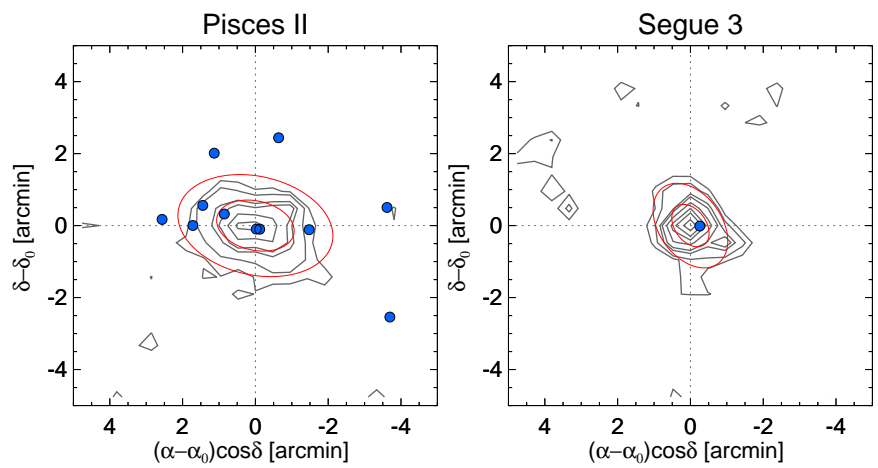

FIG. 3.- Density contours (black) of candidate member stars selected from the KPNO data centered on each satellite. Left: Pisces II is mapped out with RGB stars. Right: Segue 3 is mapped out with MS stars. Contour levels are 3, 5, 8, 10, $15 \sigma$ (also 20 and $30 \sigma$ for Segue 3) above the background. Red ellipses show Plummer isodensity contours corresponding to one and two halfradii. Blue dots mark the locations of the BHB candidate stars.

Pisces II - The KPNO photometry reaches at least 2 magnitudes fainter than the SDSS and plays a crucial role in the identification and analysis of Pisces II, as can be seen from Fig. 2, The stellar over-density is enhanced, with a significance of $\sim 8$. The CMD is now quite unambiguous, with both RGB and BHB clearly visible together with a pile-up of stars around the main sequence turn-off (MSTO). The very tight BHB can now be used to estimate the distance modulus of the system $(\mathrm{m}-\mathrm{M})_{0}=21.3$. Both CMD and the Hess difference panels of Fig. 2 show over-plotted the ridge-lines of the globular clusters M92 and M13 from Clem (2005). The RGB stars in Pisces II seem to be described equally well by both and, hence, are likely to possess metallicity in
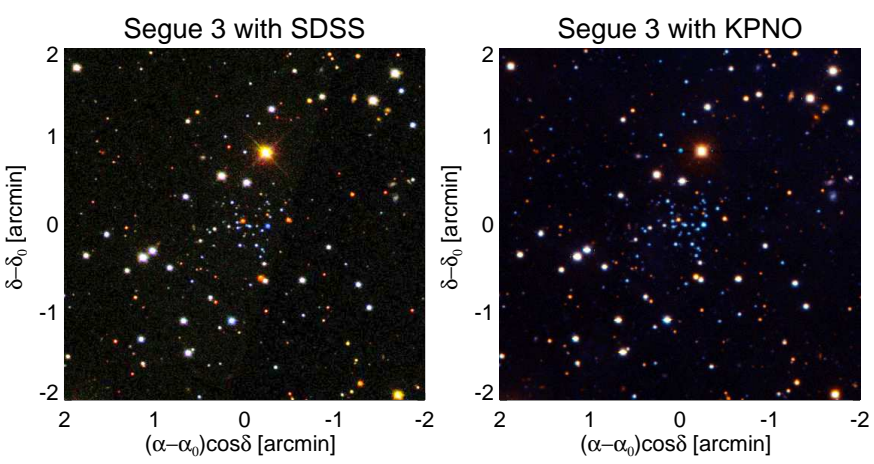

FIG. 4.- Color images covering $4^{\prime} \times 4^{\prime}$ region centered on Segue 3 made with SDSS (left) and KPNO (right) data. SDSS image is made with $g, r$ and $i$ band frames. KPNO image is made with $g$ and $r$ band frames.

the range of $-2.3<[\mathrm{Fe} / \mathrm{H}]<-1.55$ (Harris 1996). Note, however, that the two brightest RGB stars are located right on top of the M13 ridgeline. To select all likely members of Pisces II, we draw a wide mask shown in the right panel of Fig. 2 we also select the $\mathrm{BHB}$ candidate stars with a color-magnitude box shown in the middle left panel. Using these selection cuts we can now measure the structural parameters of Pisces II and its luminosity. The density contours of the RGB and MSTO stars are shown in the left panel of Fig. 3. To measure the satellite's halflight radius, ellipticity and position angle, we follow the procedure outlined in Martin et al. (2008). From the locations of candidate MSTO and RGB stars we measure a half-light radius of $1 ! 1$ ( or $\sim 60$ pc at a distance of $\sim$ $180 \mathrm{kpc}$ ) with noticeable ellipticity. The results of this maximum likelihood fit are reported in Table 1

To estimate the total luminosity of Pisces II, we i) in- 
tegrate the flux inside the mask shown in the right-most panel of Figure 2 within $3^{\prime}$ to get $V=16.6 \mathrm{mag}$; ii) subtract an estimate of background contamination of 17.4 mag; iii) add the contribution from the BHBs and possible blue stragglers (BS) of $\mathrm{V}=19.4$ to get $M_{\mathrm{V}}=-4.2$. To account for the missing flux in the fainter stars, we use the luminosity function of the Ursa Minor dSph, which we integrate within $3.7<\mathrm{V}<13$ to get $0.8 \mathrm{mag}$. This can be compared to $0.5 \mathrm{mag}$ correction if the luminosity function of a typical globular cluster is used (see e.g. Niederste-Ostholt et al. (2010)). Out of all ingredients contributing to the total luminosity, the exact number of RGB members of Pisces II carries the largest uncertainty. Our final estimate of the luminosity of Pisces II is $M_{\mathrm{V}}=-5 \pm 0.5$ mag.

Segue 3-This satellite is one of the very few recent discoveries that can be seen seen directly in the SDSS images. Figure 4 shows color images of $4^{\prime} \times 4^{\prime}$ area centered on Segue 3 made with $g, r$ and $i$ SDSS frames and $g$ and $r$ KPNO frames. SDSS and KPNO data are of comparable quality, but the KPNO frames are integrated longer and hence the faint objects are detected and measured with greater accuracy. On both images, a central concentration of bluish stars belonging to Segue 3 can be seen, albeit with a somewhat irregular distribution. The KPNO photometry presented in the lower panel of Figure 2 reveals a rather tight main sequence, with a clear turn-off, which we use to estimate the distance modulus of $(\mathrm{m}-\mathrm{M})_{0}=16.1$. Of the two ridgelines over-plotted on the satellite's CMD, M92, with $[\mathrm{Fe} / \mathrm{H}] \sim-2.3$, clearly provides a better match. We use the KPNO data to derive the structural parameters of Segue 3 by following the procedure mentioned above. The half-light radius of Segue 3 is 0.65 and, overall, its distribution of stars is circular (see Table 11), although there are some irregularities in the density profiles as can be seen from the right panel of Figure 3 most likely due to the small numbers of stars. We calculate the luminosity of Segue 3 in two steps, using both SDSS and KPNO data. For the MS members fainter than $r=19$, we integrate the flux within $2^{\prime}$ inside the mask shown in the bottom right panel of Figure 2 and subtract the estimate of the background contamination (outside $2^{\prime}$ ) to get $\mathrm{V}=16.6$. For the $\mathrm{RGB}$ members $(r<19)$, we integrate flux inside a narrower mask shown in the middle-left panel of Figure 1, which, after the background subtraction, gives $\mathrm{V}=15.15$. So, the total $\mathrm{V}=14.9$, or $M_{\mathrm{V}}=-1.2$. This is accurate to not less than $0.5 \mathrm{mag}$ and can be better constrained when the true RGB members are identified through spectroscopic follow-up.

\section{DISCUSSION AND CONCLUSIONS}

Pisces II is close on the sky to Pisces I, discovered as an overdensity of RR Lyraes by Watkins et al. (2009) and confirmed spectroscopically by Kollmeier et al. (2009). But, at a heliocentric distance of $\sim 180 \mathrm{kpc}$, Pisces II is almost twice as far as away. The extent of Pisces I is probably considerable, at least as judged from the RR Lyrae populations (see Figure 12 of Watkins et al. 2009). This might imply the break-up of a substantial satellite galaxy moving on a radial orbit, in which case it is natural to interpret Pisces II as a further fragment or companion. Pisces II is very similar in morphology, size and luminosity to a number of recent discoveries such as Hercules, Leo IV and Leo V (Belokurov et al. 2007a, 2008). They also all lie at similar heliocentric distances of $\sim 150 \mathrm{kpc}$. All four have extended BHB populations enshrouding them.

Segue 3 is a very close relative of Koposov 1 and 2 , the ultrafaint star clusters at distances of $\sim 50$ kpc (Koposov et al. 2007). All three objects have a similar size $(\sim 3 \mathrm{pc})$, luminosity $M_{\mathrm{V}} \sim-1$ and contain only a few tens of stars. Unlike Koposov 1 and 2 , Segue 3 is much closer, and might even be a part of the Hercules-Aquila Cloud (Belokurov et al. 2007b; Watkins et al. 2009). The evolution of such objects is known to proceed with pronounced mass segregation. The very few heavy stars sink to the centre, and the lighter stars are ejected to form a diffuse corona. It would be interesting to verify this prediction with spectrocopic surveys of the objects.

Funding for the SDSS and SDSS-II has been provided by the Alfred P. Sloan Foundation, the Participating Institutions, the National Science Foundation, the U.S. Department of Energy, the National Aeronautics and Space Administration, the Japanese Monbukagakusho, the Max Planck Society, and the Higher Education Funding Council for England. The SDSS Web Site is http://www.sdss.org/. VB thanks the Royal Society for financial support. SK, MAW, LLW and LW all thank the Science and Technology Facilities Council of the UK for funding. EO acknowledges NSF grant AST-0807498; MM acknowledges NSF grant AST-0808043

\section{REFERENCES}

\author{
Abazajian, K. N., et al. 2009, ApJS, 182, 543 \\ Belokurov, V. et al. 2006, ApJ, 647, L111 \\ Belokurov, V. et al. 2007a, ApJ, 654, 897 \\ Belokurov, V., et al. 2007b, ApJ, 657, L89 \\ Belokurov, V., et al. 2008, ApJ, 686, L83 \\ Belokurov, V., et al. 2009, MNRAS, 397, 1748 \\ Clem, J. L. 2005, PhD Thesis, University of Victoria \\ Fukugita, M., Ichikawa, T., Gunn, J. E., Doi, M., Shimasaku, K., \\ \& Schneider, D. P. 1996, AJ, 111, 1748 \\ Gunn, J.E. et al. 1998, AJ, 116, 3040 \\ Gunn, J.E. et al. 2006, ApJ, in press \\ Harris, W. E. 1996, AJ, 112, 1487 \\ Irwin, M. J., et al. 2007, ApJ, 656, L13 \\ Irwin, M.J., \& Lewis, J. 2001, New Astronomy Review, 45, 105 \\ Jannuzi, B. T., Dey, A., Tiede, G. P., Brown, M. J. I., \& NDWFS \\ Team 2000, Bulletin of the American Astronomical Society, 32, \\ 1528 \\ Kollmeier, J. A., et al. 2009, ApJ, 705, L158
}

Koposov, S., et al. 2007, ApJ, 669, 337

Koposov, S., et al. 2008, ApJ, 686, 279

Lupton, R., Gunn, J., \& Szalay, A. 1999, AJ, 118, 1406

Martin, N. F., de Jong, J. T. A., \& Rix, H.-W. 2008, ApJ, 684, 1075

Niederste-Ostholt, M., Belokurov, V., Evans, N. W., Gilmore, G., Wyse, R. F. G., \& Norris, J. E. 2009, MNRAS, 398, 1771

Niederste-Ostholt, M., Belokurov, V., Evans, N. W., Peñarrubia,

J., 2010, ApJ, submiited, arxiv/1002.0266

Schlegel, D. J., Finkbeiner, D. P., \& Davis, M. 1998, ApJ, 500 525

Smith, J. A., et al. 2002, AJ, 123, 2121

Stoughton, C. et al. 2002, AJ, 123, 485

Walsh, S. M., Jerjen, H., \& Willman, B. 2007, ApJ, 662, L83

Watkins, L. L., et al. 2009, MNRAS, 398, 1757

Willman, B., et al. 2005, ApJ, 626, L85

Yanny, B., et al. 2009, AJ, 137, 4377

Zucker, D. B., et al. 2006a, ApJ, 643, L103 
Zucker, D. B., et al. 2006b, ApJ, 650, L41 